\title{
Dispersion relation of flow-induced wave of a flexible web subjected to fluid flow in a narrow gap
}

\author{
M. Watanabe, G. Kudou \& K. Hara \\ Department of Mechanical Engineering, Aoyama Gakuin University, \\ Japan
}

\begin{abstract}
This paper deals with a theoretical stability analysis of a flow-induced wave generated in a flexible web subjected to fluid flow in a narrow gap. The flexible web is subjected to both fluid flow and in-plane tensile force. The flow-induced wave occurs to the flexible web resulting from fluid-structure interaction between out-of-plane motion of the flexible web and the fluid flow. The effect of the fluid flow and tensile force on the stability of the wave is examined. The theoretical results show that traveling-wave type unstable wave occurs to the flexible web due to the fluid flow in the narrow gap, and clarify the dispersion relation of the flow-induced wave of the web, i.e., phase velocity (traveling-wave speed) and growth rate of the wave, which are as a function of the flow velocity and tensile force. The theoretical results are verified by experiments on the most unstable wave number and critical flow velocity.
\end{abstract}

Keywords: flow-induced wave, flexible web, dispersion relation, fluid-structure interaction.

\section{Introduction}

In the manufacturing process of flexible materials, such as a paper, sheet and plastic films, the flexible materials are subjected to a fluid flow in a narrow gap for non-contact support and drying. However the interaction of the thin film with the fluid flow causes an unstable wave. The flow-induced wave reduces the process efficiency and generates noise, in some case, causes fatigue failure to the thin film. 
Up to this time, many studies on the stability of leakage-flow-induced vibrations and sheet flutter have been reported. Inada and Hayama [1, 2] studied leakage-flow-induced vibrations of translational one-degree-of-freedom system, and clarified the instability mechanism of these systems theoretically. Nagakura and Kaneko [3] examined the stability of a cantilever beam subjected to the leakage fluid flow by employing the modal analysis method, and they verified the theoretical results comparing with experimental results.

Some studies on the flow induced vibration and flow induced wave of a flexible sheet have been reported [4-9]. Chang and Moretti [8] studied flow induced vibration of free edges of thin films by using a traveling-wave analysis based on the incompressible potential-flow. In their study, previous studies of edge flutter on two-dimensional waves expands to a more realistic threedimensional problem, and the simple closed form solutions of the critical flow velocity are presented. Watanabe and Kobayashi [9] studied the stability and dynamic characteristics of a moving flexible sheet with shear fluid flow. In their study, unstable wave occurs to the flexible sheets as a type of traveling-wave due to the shear fluid flow.

This paper presents the theoretical stability analysis of the flow-induced wave generated in the web subjected to the fluid flow in the narrow gap. In the stability analysis, tensile force is applied to the web, and the web is subjected to air flow in a narrow gap. The analytical results show the stability and wave characteristics of the flow-induced wave, phase velocity and growth rate of the wave.

\section{Theoretical stability analysis}

\subsection{Modeling and coordinate system}

Fig. 1 and Fig. 2 show a traveling wave motion and an analytical model of the web subjected to the fluid flow together with the stationary coordinate system $x y z$ and geometrical parameters considered in this model. The web is set in the middle of the narrow gap filled with fluid and is subjected to the fluid flow in $y$ direction. Tensile force is applied to the web. The equilibrium gap width in steady state is a constant, $H_{0}$, and thickness of the web is $h$. The uniform tensile

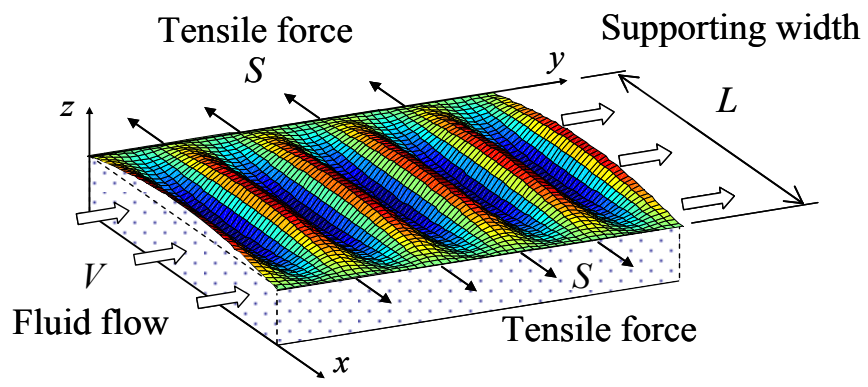

Figure 1: $\quad$ Traveling wave motion generated in the web (thin film). 
force per unit width $S$ is applied in $x$-direction. The fluid flow and tensile force are crossed at a right angle.

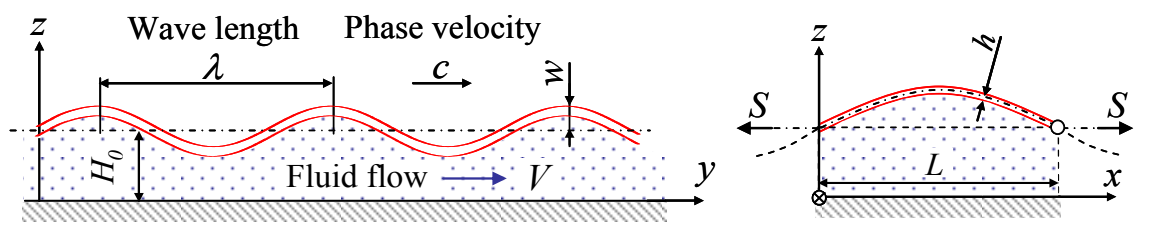

Figure 2: $\quad$ Analytical model of the flow-induced wave.

\subsection{Basic equation of structural motion and fluid flow}

The equation of motion of the web subjected to the fluid flow is derived in terms of the transverse displacement $w$ and with respect to the stationary coordinate system $x y z$ as follows:

$$
\rho h \frac{\partial^{2} w}{\partial t^{2}}+C_{s} \frac{\partial w}{\partial t}+-S \frac{\partial^{2} w}{\partial x^{2}}+\frac{h^{3}}{12}\left(E+E^{*} \frac{\partial}{\partial t}\right)\left(\frac{\partial^{4} w}{\partial x^{4}}+2 \frac{\partial w^{4}}{\partial x^{2} \partial y^{2}}+\frac{\partial^{4} w}{\partial y^{4}}\right)=f,
$$

where $E$ is Young's modulus, $C_{s}$ and $E^{*}$ are the external and internal structural damping coefficients, respectively. $f$ is fluid force acting on the web surface per unit area. $\rho$ and $h$ are the density and thickness of the thin film, respectively.

The equations of motion of the fluid flow around the web are derived by integrating the continuity and momentum equations of fluid motion over the gap width $H_{0}$ as follows:

$$
\begin{gathered}
\frac{\partial H}{\partial t}+\frac{\partial}{\partial y}(H V)=0 \\
\frac{\partial}{\partial t}(H V)+\frac{\partial}{\partial y}\left(H V^{2}\right)+\frac{H}{\rho_{f}} \frac{\partial P}{\partial y}+\frac{12 \mu}{\rho_{f}}\left(V-V_{0}\right)-\frac{1}{2}\left(f_{s}-f_{w}\right) V^{2}=0
\end{gathered}
$$

where $V$ is mean flow velocity over the gap width between the web and side wall. $V_{0}$ is steady flow velocity of the fluid flow in the narrow passage. $\mu$ is fluid viscosity. $f_{s}$ and $f_{w}$ are fluid friction coefficients on the surface of the web and side wall, respectively, and are assumed to be obtained as follows [10]:

$$
f_{s}=f_{w}=\frac{1}{4}\left\{1.14-2 \log _{10}\left(\frac{\varepsilon_{H}}{D_{H}}+\frac{21.25}{\mathrm{Re}^{0.9}}\right)\right\}^{-2},
$$

where the friction coefficients $f_{s}$ and $f_{w}$ depend on the Reynolds number Re and the roughness of surface $\varepsilon_{H}$. The Reynolds number is defined as $\operatorname{Re}=V D_{H} / v_{f}$, where $D_{H}=2 H_{0}, \mu_{f}$ is kinematic viscosity of the fluid and $\varepsilon_{H}$ is roughness of the side wall respectively. 


\subsection{Wave equation}

In this section, a linear wave equation of bending wave motion of the web coupled with the fluid flow in the narrow gap is derived from the basic equations of motion of the web and fluid flow.

The gap width $H$ between the web and side wall is obtained using the steady equilibrium gap width $H_{0}$ and transverse displacement $w$ of the web as follows:

$$
H(x, y, t)=H_{0}+w(x, y, t) .
$$

Similarly, the flow velocity and fluid pressure are obtained by summation of the steady and unsteady terms as follows:

$$
\begin{gathered}
V(y, t)=V_{0}+\Delta v(y, t), \\
P(x, y, t)=P_{0}(y)+p(x, y, t),
\end{gathered}
$$

where $V_{0}$ and $\Delta v$ are steady and unsteady flow velocities, and $P_{0}$ and $p$ are steady and unsteady fluid pressures, respectively. Here the unsteady fluid pressure is induced by the motion of the web.

Substituting eqn. (5), (6) and (7) into the basic eqn. (2) and (3), the basic equations of the fluid flow are linearized with respect to the unsteady terms as follows:

$$
\begin{gathered}
\frac{\partial \hat{w}}{\partial T}+\frac{\partial \Delta \hat{v}}{\partial Y}+\hat{V} \frac{\partial \hat{w}}{\partial Y}=0, \\
\frac{\partial \Delta \hat{v}}{\partial T}+\hat{V} \frac{\partial \hat{w}}{\partial T}+2 \hat{V} \frac{\partial \Delta \hat{v}}{\partial Y}+\hat{V}^{2} \frac{\partial \hat{w}}{\partial Y}+\frac{12 \hat{\mu} \hat{h}}{\hat{\rho}} \Delta \hat{v} \\
+\left(f_{w}-f_{s}\right) \hat{V} \Delta \hat{v}+\frac{1}{2}\left(f_{s}-f_{w}\right) \hat{V}^{2} \hat{w}+\frac{\hat{h}}{\hat{\rho}} \frac{\partial \hat{p}}{\partial Y}=0,
\end{gathered}
$$

where each term is expressed by dimensionless parameters by defining the following quantities:

$$
\begin{gathered}
T_{0}=\sqrt{\frac{\rho h H_{0}^{4}}{D}}, U_{0}=\frac{H_{0}}{T_{0}}, T=\frac{t}{T_{0}}, \hat{V}=\frac{V_{0}}{U_{0}}, \hat{L}=\frac{L}{H_{0}}, \hat{w}=\frac{w}{H_{0}}, \\
X=\frac{x}{H_{0}}, Y=\frac{y}{H_{0}}, \hat{h}=\frac{h}{H_{0}}, \Delta \hat{v}=\frac{\Delta v}{U_{0}}, \hat{\rho}=\frac{\rho_{f}}{\rho}, \hat{S}=\frac{S H_{0}^{2}}{D}, \hat{p}=\frac{H_{0}^{3}}{D} p, \\
\hat{\mu}=\mu \sqrt{\frac{H_{0}^{2}}{\rho h D}}, \hat{C_{s}}=C_{s} \sqrt{\frac{H_{0}^{4}}{\rho h D}}, \hat{f}=\frac{H_{0}^{3}}{D} f, \varepsilon=\frac{E}{E T_{0}}, D=\frac{E h^{3}}{12} .
\end{gathered}
$$

Similarly, the dimensionless basic equation of the web is obtained from eqn. (1) using the dimensionless parameters as follows:

$$
\frac{\partial^{2} \hat{w}}{\partial T^{2}}+\hat{C}_{s} \frac{\partial \hat{w}}{\partial T}-\hat{S} \frac{\partial^{2} \hat{w}}{\partial X^{2}}+\left(1+\varepsilon \frac{\partial}{\partial T}\right)\left(\frac{\partial^{4} \hat{w}}{\partial X^{4}}+2 \frac{\partial^{4} \hat{w}}{\partial X^{2} \partial Y^{2}}+\frac{\partial^{4} \hat{w}}{\partial Y^{4}}\right)=\hat{f}(X, Y, T),
$$


where $\hat{f}$ denotes the dimensionless fluid force obtained from the dimensionless unsteady fluid pressure $\hat{p}$ using following relationship:

$$
\hat{f}(X, Y, T)=\hat{p}(X, Y, T) .
$$

From these linearized equations, eqn. (8), (9) and (11), a linear wave equation of bending wave motion of the web coupled with the fluid flow is obtained as follows:

$$
\begin{aligned}
& \left(\frac{\partial}{\partial T}+\hat{V} \frac{\partial}{\partial Y}\right)^{2} \hat{w}+\gamma\left(\frac{\partial}{\partial T}+\frac{\partial}{\partial Y}\right) \hat{w} \\
& =\frac{1}{\beta} \frac{\partial^{2}}{\partial Y^{2}}\left\{\frac{\partial^{2} \hat{w}}{\partial T^{2}}+\hat{C}_{s} \frac{\partial \hat{w}}{\partial T}-\hat{S} \frac{\partial^{2} \hat{w}}{\partial X^{2}}+\left(1+\varepsilon \frac{\partial}{\partial T}\right)\left(\frac{\partial^{4} \hat{w}}{\partial X^{4}}+2 \frac{\partial^{4} \hat{w}}{\partial X^{2} \partial Y^{2}}+\frac{\partial^{4} \hat{w}}{\partial Y^{4}}\right)\right\},
\end{aligned}
$$

where $\beta, \gamma$ and $\delta$ are defined by the following dimensionless parameters:

$$
\beta=\frac{\hat{\rho}}{\hat{h}}, \quad \gamma=\frac{12 \hat{\mu} \hat{h}}{\hat{\rho}}+\left(f_{w}-f_{s}\right) \hat{V}, \delta=\hat{V}-\frac{\alpha}{\gamma},
$$

where $\alpha$ is defined by the following dimensionless parameters:

$$
\alpha=\frac{1}{2}\left(f_{s}-f_{w}\right) \hat{V}^{2} .
$$

From eqn. (13), it is found that the wave equation is composed two fluidwave equations expressed by first and second-order differential equations and the structural wave equation derived from the equation of motion of the web. The first fluid-wave equation is derived from the inertia term of the basic equation of the web and the continuity equation of the fluid flow. The second fluid-wave equation is derived from the viscosity and friction loss terms of the basic equation of the web and the continuity equation of the fluid flow.

Moreover, in eqn. (13), $\gamma$ denotes a dimensionless parameter of the viscosity and friction loss of the fluid. $\beta$ denotes a dimensionless parameter which has the effect of the fluid-waves on the structural wave of the web. If $\beta$ is large, the fluid-waves are dominant compared with the structural wave traveling in the web. On the other hand, if $\beta$ is small, the structural wave is dominant compared with the fluid-waves. Thus, it is found that the dimensionless parameter $\beta$ is one of the most important parameter for the dynamic characteristics and stability of the wave.

\subsection{Dispersion relation and stability analysis}

In this section, the dispersion relation of the wave is derived from the wave equation, eqn.(13), to investigate the dynamic characteristics, phase velocity (traveling-wave velocity) and growth rate of the wave. The transverse displacement $w$ of the web is expressed as follows:

$$
\hat{w}=\hat{w}_{0} \sin \left(\frac{\pi X}{\hat{L}}\right) \exp \{i(\hat{\omega} T-k Y)\} .
$$


Substituting eqn. (16) into the wave eqn. (13), the dispersion relation of the wave is obtained as follows:

$$
\begin{aligned}
\left(\beta+k^{2}\right) \hat{\omega}^{2}-2 k \beta \hat{V} \hat{\omega}-\left[\gamma \beta+\hat{C}_{s} k^{2}+k^{2} \varepsilon\left\{\left(\frac{\pi}{\hat{L}}\right)^{2}+k^{2}\right\}^{2}\right] i \hat{\omega} \\
+\left[\beta k^{2} \hat{V}^{2}-k^{2}\left\{\left(\frac{\pi}{\hat{L}}\right)^{2}+k^{2}\right\}^{2}-\hat{S} k^{2}\left(\frac{\pi}{\hat{L}}\right)^{2}\right]+i \gamma \beta \delta k=0 .
\end{aligned}
$$

The stability and dynamic characteristics of the wave generated in the web are dependent on the complex root, $\hat{\omega}$ obtained from eqn. (17), where $\operatorname{Re}[\hat{\omega}] / k$, real part of $\hat{\omega}$ divided wave number $k$, is denotes the phase velocity of the wave. If $\operatorname{Re}[\hat{\omega}] / k$ is positive, the wave is forward wave which propagates in the same direction as the fluid flow, and if $\operatorname{Re}[\hat{\omega}] / k$ is negative, the wave is backward wave which propagates in the opposite direction as the fluid flow. Moreover, $-\operatorname{Im}[\hat{\omega}]$, negative imaginary part of $\hat{\omega}$, denotes the growth rate of the wave. If $-\operatorname{Im}[\hat{\omega}]$ is positive, the wave grows exponentially in time, that is, an unstable wave occurs, and if $-\operatorname{Im}[\hat{\omega}]$ is negative, the wave is stable.

\section{Analytical parameters}

Table 1 shows parameters used in the calculation for the stability analysis. These parameters correspond to the experimental parameters used in the other experimental study. In the calculations, the web is thin film and is made of PET, and the fluid in the gap between the web and side wall is air. Moreover, the external and internal structural damping coefficient are assumed $C_{s}=10.0 \mathrm{Ns} / \mathrm{m}^{3}$ and $E^{*}=1.0 \times 10^{3} \mathrm{Ns} / \mathrm{m}^{2}$. In this conditions, dimensionless parameters are $\beta=2.46 \times 10^{-1}, \hat{C}_{s}=1.65 \times 10^{-2}, \varepsilon=9.01 \times 10^{-5}$ and $\hat{S}=2.40 \times 10^{3}$.

Table 1: $\quad$ Parameters used in the calculation.

\begin{tabular}{|c|c||c|c|}
\hline Material of the web & PET & Tensile force $S[\mathrm{~N} / \mathrm{m}]$ & $50 \sim 200$ \\
\hline Fluid & Air & Young's modulus $E[\mathrm{~Pa}]$ & $3.0 \times 10^{9}$ \\
\hline Gap width $H_{0}[\mathrm{~m}]$ & $5.0 \times 10^{-3}$ & Web density $\rho\left[\mathrm{kg} / \mathrm{m}^{3}\right]$ & $1.4 \times 10^{3}$ \\
\hline Web width $L[\mathrm{~m}]$ & $0.3 \sim 0.6$ & Fluid density $\rho_{f}\left[\mathrm{~kg} / \mathrm{m}^{3}\right]$ & 1.1 \\
\hline Web thickness $h[\mathrm{~m}]$ & $16.0 \times 10^{-6}$ & Fluid viscosity $\mu\left[\mathrm{Ns} / \mathrm{m}^{2}\right]$ & $18.2 \times 10^{-6}$ \\
\hline
\end{tabular}

\section{Analytical results}

\subsection{Phase velocity and growth rate of wave}

Fig. 3 (a) and (b) show the phase velocity $\operatorname{Re}[\hat{\omega}] / k$ and growth rate $-\operatorname{Im}[\hat{\omega}]$ of the wave generated in the web subjected to the fluid flow in the narrow passage 
as a function of the flow velocity $\hat{V}$. In this figure, three types of curve denote the results of wave number $k=1.2,1.6,2.0$, respectively. From Fig. 3(a), it is seen that there are forward and backward waves for each wave number at the flow velocity of zero, and vary with increasing flow velocity. The phase velocity of the backward wave progressively goes to zero. After the phase velocity of the backward wave has become zero, the backward wave becomes forward wave, and its phase velocity increases as the flow velocity increases. From Fig. 3(b), it is seen that unstable (flow-induced) waves occurs because growth rate of the forward wave becomes positive, and that the waves occur as a type of traveling wave.

(a)

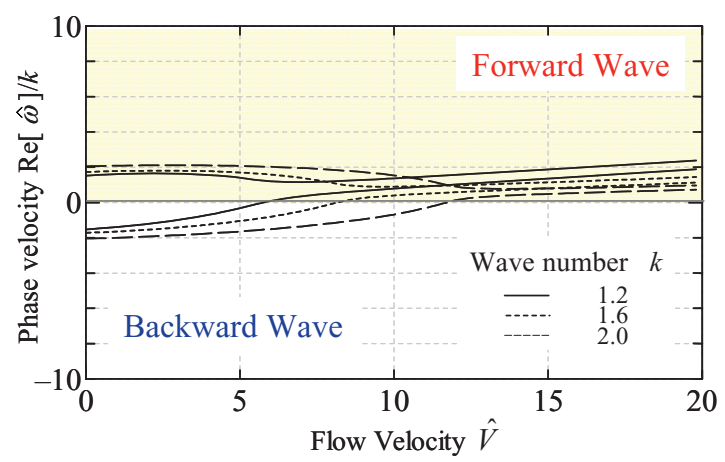

(b)

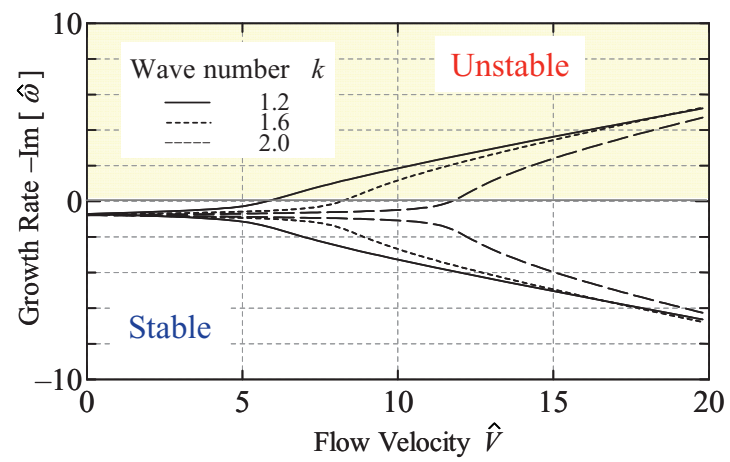

Figure 3: Phase velocity $\operatorname{Re}[\hat{\omega}] / k$ and growth rate $-\operatorname{Im}[\hat{\omega}]$ with changing flow velocity $\hat{V}$.

\subsection{Unstable region}

Fig. 4(a) shows the unstable region of the wave with changing the flow velocity as a function of the tensile force $\hat{S}$. In this figure, four types of curve denote the result of the tensile force $\hat{S}=1.2 \times 10^{3} \sim 4.8 \times 10^{3}$. It can be seen that the unstable region of wave number becomes narrower with increasing the tensile force, and 
the critical flow velocity increases with increasing the tensile force. The unstable region of the wave number becomes broader with increasing the fluid flow. Moreover, it can be seen that the most unstable wave number is about $k=0.5$. Fig. 4(b) shows the unstable region of the wave with changing the flow velocity as a function of the web supporting width. In this figure, four types of curve denote the results of the web supporting width $\hat{L}=60 \sim 120$. It can be seen that the unstable region of wave number becomes broader with increasing the web supporting width, and the critical flow velocity decreases with increasing the web supporting width. The unstable region of the wave number becomes broader with increasing the flow velocity.

(a)

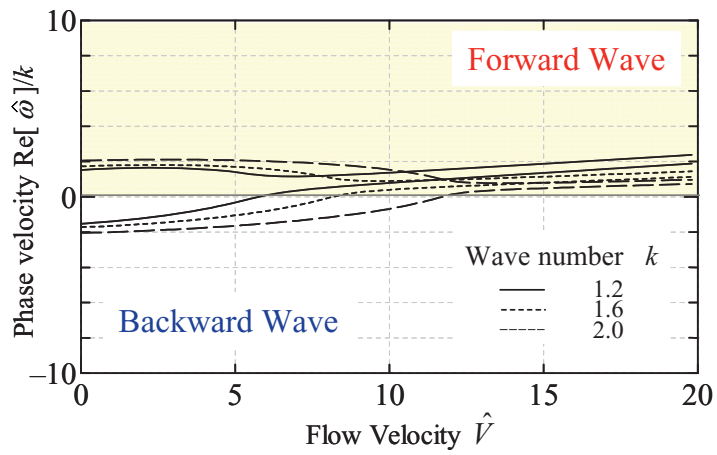

(b)

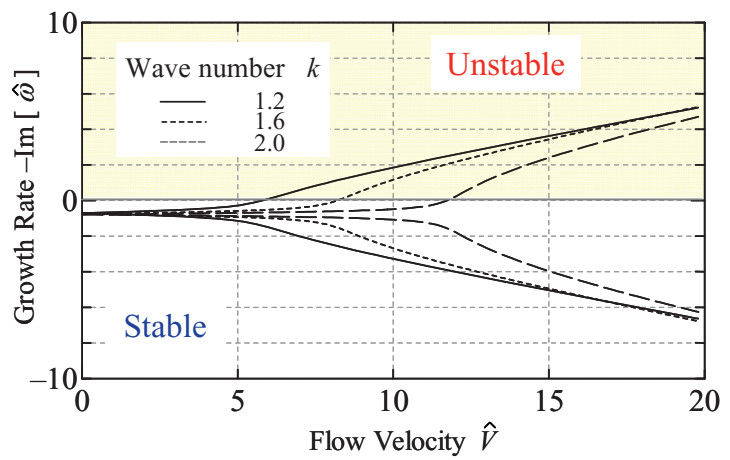

Figure 4: Unstable region with changing tensile force applied to the web $\hat{S}$ and supporting width $\hat{L}$.

\section{Conclusions}

The theoretical stability analysis of the flow-induced wave generated in the web subjected to the fluid flow in the narrow gap was developed. In the stability analysis, the effect of the fluid flow, tensile force, and web supporting width on the stability were examined and the dispersion relation of the flow-induced wave were clarified. The following main conclusions were obtained: 
(1) The flow-induced traveling wave occurs in the web due to the fluid flow in the narrow gap when the flow velocity becomes higher.

(2) The unstable region of wave number becomes narrower with increasing the tensile force, and becomes broader with increasing the flow velocity. The critical flow velocity increases with increasing the tensile force.

(3) The unstable region of wave number becomes broader with increasing the web supporting width, and the critical flow velocity decreases with increasing the web supporting width.

(4) The flow-induced wave is generated in which the wave number is about $k=0.5$.

\section{References}

[1] F. Inada and S. Hayama, A Study on Leakage-Flow-Induced Vibrations (1st Report, Fluid-Dynamic Forces Acting on the Walls of a One-Dimensional, Narrow, Tapered Passage), JSME Int. J. Series III, 31, pp.39-47, 1998.

[2] F. Inada and S. Hayama, A Study on Leakage-Flow-Induced Vibrations. Part 1: Fluid-Dynamic Forces and Moments Acting on the Walls of a Narrow Tapered Passage, Journal of Fluids and Structures, 4, pp.395-412, 1990.

[3] H. Nagakura and S. Kaneko, The Stability of a Cantilever Beam Subjected to One-Dimensional Leakage Flow (in Japanese), Transactions of the JSME. Series C, 58 (546), pp.352-259, 1992.

[4] C. A. Evrensel and A. Kalnins, Response of Compliant Slab to Inviscid Incompressible Fluid Flow, Journal of Acoustical Society of America, 78, pp.2034-2041, 1985.

[5] R. J. Hansen and D. J. Huston, C. C. Ni, An Experimental Study of FlowInduced Waves on a Flexible Surface, Journal of Sound and Vibration, 68, pp.317-334, 1980.

[6] P. W. Carpenter and A.D.Garrad, The Hydrodynamic Stability of Flow Over Kramer-Type Compliant Surface. Part 1. Tollmien-Schlichting Instabilities, Journal of Fluid Mechanics, 155, pp.465-510, 1985.

[7] P. W. Carpenter and A. D. Garrad, The Hydrodynamic Stability of Flow Over Kramer-Type Compliant Surface. Part 2. Flow-Induced Surface Instabilities, Journal of Fluid Mechanics, 170, pp.199-232, 1986.

[8] Y. B .Chang and P. M. Moretti, Flow-Induced Vibration of Free Edges of Thin Film, Journal of Fluids and Structures, 16, pp.989-1008, 2002.

[9] M. Watanabe and N. Kobayashi, Dynamics and Stability Analysis of Axially Moving Web Subjected to Shear Fluid Flow in Narrow Space (in a Case of Finitely Long Space) (in Japanese), Transaction of the JSME. Series C, 67(664), pp.3722-3729, 2001.

[10] F. Axisa and J. Antunes, Flexural Vibrations of Rotors Immersed in Dense Fluids Part I, Theory, Journal of Fluids and Structures, 6, pp.3-21, 1992 